\title{
ON THE EXISTENCE OF A NONTRIVIAL EQUILIBRIUM IN RELATION TO THE BASIC REPRODUCTIVE NUMBER
}

\author{
KARUnia PUtra WiJAYA ${ }^{a, *}$, SUTIMIN $^{b}$, Edy SOEWONO $^{c}$, ThOMAS GÖTZ $^{a}$ \\ ${ }^{a}$ Mathematical Institute \\ University of Koblenz, Universität Straße 1, 56070 Koblenz, Germany \\ e-mail: karuniaputra@uni-koblenz.de \\ ${ }^{b}$ Department of Mathematics \\ Diponegoro University, Jalan Prof. H. Soedarto, SH, 50275 Semarang, Indonesia \\ ${ }^{c}$ Department of Mathematics \\ Bandung Institute of Technology, Jalan Ganesha 10, 40132 Bandung, Indonesia
}

\begin{abstract}
Equilibrium analysis in autonomous evolutionary models is of central importance for developing long term treatments. This task typically includes checks on the existence and stability of some equilibria. Prior to touching on the stability, one often attempts to determine the existence where the basic reproductive number $\mathcal{R}_{0}$ plays a critical role as a threshold parameter. When analyzing a nontrivial equilibrium (e.g., an endemic, boundary, or coexistence equilibrium) where $\mathcal{R}_{0}$ is explicit, we usually come across a typical result: if $\mathcal{R}_{0}>1$, then a nontrivial equilibrium exists in the biological sense. However, for more sophisticated models, $\mathcal{R}_{0}$ can be too complicated to be revealed in terms of the involving parameters; the task of relating the formulation of a nontrivial equilibrium to $\mathcal{R}_{0}$ thus becomes intractable. This paper shows how to mitigate such a problem with the aid of functional analysis, adopting the framework of a nonlinear eigenvalue problem. An equilibrium equation is first to be transformed into a canonical equation in a lower dimension, and then the existence is confirmed under several conditions. Three models are tested showing the applicability of this approach.
\end{abstract}

Keywords: autonomous model, nontrivial equilibrium, basic reproductive number, nonlinear eigenvalue problem.

\section{Introduction}

Guaranteeing the existence of a nontrivial equilibrium in relation to the basic reproductive number $\mathcal{R}_{0}$, especially within small-sized autonomous models, is easy since $\mathcal{R}_{0}$ is explicit. For example, the basic reproductive number of the following SIR model:

$$
\begin{aligned}
\dot{S} & =\mu(N-S)-\frac{\beta}{N} S I+\kappa R, \\
\dot{I} & =\frac{\beta}{N} S I-(\gamma+\mu) I, \\
\dot{R} & =\gamma I-(\kappa+\mu) R,
\end{aligned}
$$

where $N:=S+I+R$ is constant, is given by $\mathcal{R}_{0}=\beta /$ $(\gamma+\mu)$. How we arrive at this formula will be explained shortly. Simple algebraic substitutions reveal the so-called

\footnotetext{
*Corresponding author
}

endemic equilibrium,

$$
\left(\frac{N}{\mathcal{R}_{0}}, \frac{(\kappa+\mu)\left(\mathcal{R}_{0}-1\right) N}{(\gamma+\kappa+\mu) \mathcal{R}_{0}}, \frac{\gamma\left(\mathcal{R}_{0}-1\right) N}{(\gamma+\kappa+\mu) \mathcal{R}_{0}}\right),
$$

whose existence in the biological sense is confirmed if $\mathcal{R}_{0}>1$.

Now let us ask the following questions. What happens if the model is extended to become so complicated, e.g., with more dimension or variables, that $\mathcal{R}_{0}$ can no longer be represented explicitly in terms of parameters? Does the existence still hold for $\mathcal{R}_{0}>1$ ? If so, does the corresponding nontrivial equilibrium preserve positivity in all components? Does the existence always hold for every autonomous model? Relevant samples related to these questions that can be of consideration are, e.g., epidemic models including many patches (Arino et al., 2005) and many virus strains (Aguiar et al., 2013).

In this communication, we present several materials 
from functional analysis that help boost the work on relating the existence of a nontrivial equilibrium to the basic reproductive number in some autonomous models. In an abstract setting, the corresponding models of interest would have to be of the following fashion:

$$
\dot{\mathbf{x}}(t)=f(\mathbf{x}(t) ; \xi), \quad t \in \mathbb{R}_{+}, \mathbf{x}(0)=\mathbf{x}_{0} \in \Omega .
$$

In detail, $\mathbf{x}:[0, \infty) \rightarrow \mathbb{R}^{n}, f: \mathbb{R}^{n} \rightarrow \mathbb{R}^{n}$ are the state and the autonomous vector field of the system (1), $\xi$ is the vector of involved parameters, defined on a compact subset of $\mathbb{R}_{+}^{q}$, and $\mathbf{x}_{0}$ is the initial condition. The set $\Omega \subseteq$ $\mathbb{R}^{n}$ is a positively invariant manifold with respect to the semi-flow generated from the system (1) for a given $f$.

The first important key determinant towards successful implementation of the current framework is the ability to perform some substitutions and Taylor expansions such that the equilibrium equation

$$
f(\mathbf{x} ; \xi)=0
$$

transforms into

$$
(\mathrm{id}-\lambda L) \mathbf{x}_{I}+\omega\left(\mathbf{x}_{I}, \lambda\right)=0 \text {. }
$$

The resulting new form

$$
(\mathrm{id}-\lambda L) \mathbf{x}_{I}+\omega\left(\mathbf{x}_{I}, \lambda\right)=0
$$

is what we will repeatedly mention throughout the text as the canonical equation. The rationale behind considering (2) as such a canonic is the fact that this type of equation has appeared in some studies of nonlinear eigenvalue problems, including those we cite in this paper (Rabinowitz, 1971; 1977, Ma and Wang, 2005; Cushing, 1998). Here, $\mathbf{x}_{I} \in \mathbb{R}^{p}$ denotes another state variable of size $p$ (where $p \leq n$ ), considered to be a collection of chosen elements of $\mathbf{x}$. In epidemic models, this state variable $\mathbf{x}_{I}$ can be seen as a collection of the exposed and infective compartments. As far as (2) is concerned, $L: \mathbb{R}^{p} \rightarrow \mathbb{R}^{p}$ represents a $p \times p$ matrix whose components are of the involving parameters $\xi$ and $\omega: \mathbb{R}^{p} \times \mathbb{R} \rightarrow \mathbb{R}^{p}$ represents a vector-valued function of size $p$ of the state variable $\mathbf{x}_{I}$ and $\xi$. The new parameter $\lambda: E \rightarrow \mathbb{R}:$ $\xi \mapsto \lambda(\xi)$ is a continuous function of the parameters. The direct relationship between $\lambda$ and $\xi$ suggests to leave the arguments of $\omega$ as defined in (2); meanwhile it is assumed that

(A1) $\left\|\omega\left(\mathbf{x}_{I}, \lambda\right)\right\|=\mathcal{O}\left(\left\|\mathbf{x}_{I}\right\|^{2}\right)$ as $\mathbf{x}_{I} \rightarrow 0$

uniformly for all $\lambda$ in some compact subsets of $\mathbb{R}$. Since we are now working on finite dimensional Euclidean spaces, the corresponding norm to use is just the 2-norm, unless it is stated otherwise.

Since $\mathbf{x}_{I}=0$ solves the canonical equation (2), we call the tuples $(0, \lambda)$ for $\lambda \in \mathbb{R}$ trivial solutions. Among infinitely many trivial solutions, we are interested in a point $\left(0, \lambda_{0}\right)$ (see Definition 1) from which the canonical equation (2) bifurcates a nontrivial solution $\left(\mathbf{x}_{I}, \lambda\right)$, where $\mathbf{x}_{I} \neq 0$. In relation to the main objective in this paper, our aim is to show that by first identifying the point $\left(0, \lambda_{0}\right)$ in a close relation to the matrix $L$, conditions that certify the existence of a nontrivial (positive) solution of the canonical equation can be imposed. How $\mathcal{R}_{0}$ then comes into play is inferred when $\mathcal{R}_{0}$ is the largest eigenvalue of $L$, which we will discuss later. Together with some other apparent conditions, these give us the scope within which this approach can work.

We shall add several notes regarding the limitations of the current approach. First and foremost, this approach is meant for ODE-based autonomous models, even though it may also be valid for non-autonomous models with periodic parameters (see, e.g., Wijaya et al., 2016). The validity may be an instant check due to the similar behavior of equilibria in an autonomous model and periodic solutions in its non-autonomous counterpart, but we would rather leave it as an open research problem. Second, this approach is highly dependent on the applicability of the next generation method to determine the basic reproductive number (van den Driessche and Watmough, 2002). Therefore, this approach may not even work for simple models-classical examples are the growth model, the logistic model, and the standard Lotka-Volterra model. We would, however, refer the reader to the original paper by van den Driessche and Watmough (2002) for some epidemic models and to Wijaya et al. $(2016$; 2014) for a population dynamic model amenable to the next generation method.

We shall also mention that since this approach is concerned with the existence analysis, which never broaches the stability issue, it does not consider unwanted behavior of the model system (1) such as if it admits a solution that ceases to exist on a subset of $[0, \infty)$ or if it admits non-unique solutions. In a nutshell, the only focus of this approach is to guarantee the existence of a nontrivial solution of a given system of nonlinear equations.

\section{Background}

2.1. Basic reproductive number. In population dynamic models, the basic reproductive number $\mathcal{R}_{0}$, often referred to as the basic offspring number, determines not only the local stability of the extinction equilibrium, but also the existence of the coexistence equilibrium (Cushing, 1998; van den Driessche and Watmough, 2002; Wijaya et al., 2014; 2016). The literature reveals a crowd-pleasing definition of the basic reproductive number, when considering models for a single species, as the expected number of newborns over the course of that species's average lifetime period. In disease 
epidemics, the definition of the basic reproductive number can be extended as the number of infected individuals within an average illness period after a single infective individual interacts with its own in a completely susceptible population. The threshold value for this number is 1 , where if $\mathcal{R}_{0}<1$, then the population of individuals (or the population of infective individuals, in the case of epidemics) often evolves towards extinction, i.e., the origin. If $\mathcal{R}_{0}>1$, then the population of individuals (resp., infective individuals) evolves towards some nonzero constant vector.

In epidemiology, an equilibrium whose infective compartments are zero is known as a disease-free equilibrium, whereas that whose infective compartments are nonnegative (positive) is known as a boundary equilibrium (resp., an endemic equilibrium). For autonomous models where the sub-population of infective compartments and that of healthy compartments are separable, the basic reproductive number can be derived via the next generation method as proposed by van den Driessche and Watmough (2002). The formulation requires computation of the spectral radius of the next generation matrix, whose dimension is equal to the number of compartments in the infective sub-population. In most cases the matrix is not nilpotent (though sparse) making computation of the spectral radius nontrivial. It becomes even less possible to reveal the explicit formulation of $\mathcal{R}_{0}$ in terms of parameters if the number of infective compartments is extended to an arbitrarily high value. Technical problems do not occur until one relates the existence of a nontrivial equilibrium to the basic reproductive number $\mathcal{R}_{0}$.

Let us recall the definition of $\mathcal{R}_{0}$ based on the next generation method by first subdividing the state $\mathbf{x}$ into two sub-populations: the sub-population of individuals bringing the key characteristics of interest (growth factor or disease) $\mathbf{x}_{I}$ and the rest $\mathbf{x}_{U}$. In population dynamic models, $\mathbf{x}_{I}$ mostly serves as the whole state variables, leaving out $\mathbf{x}_{U}$ nil. In epidemic models, $\mathbf{x}_{I}$ would cover all the infective compartments (and in some models with the exposed compartments) while $\mathbf{x}_{U}$ is the collection of susceptible and recovered compartments. The disease-free equilibrium, denoted by $\mathbf{x}^{0}$, is nothing but any equilibrium of (1) where $\mathbf{x}_{I}=0$. Let $F(\mathbf{x})$ and $V(\mathbf{x})$ be respectively the vector-valued functions of new infection and of the difference between the outflows and inflows taken from the framework of the next generation method (see the work of van den Driessche and Watmough (2002) for details), with which one must have

$$
\dot{\mathbf{x}}_{I}=F(\mathbf{x})-V(\mathbf{x}) .
$$

Denote by $\mathcal{F}:=\left.\partial_{\mathbf{x}} F\right|_{\mathbf{x}=\mathbf{x}^{0}}$ and $\mathcal{V}:=\left.\partial_{\mathbf{x}} V\right|_{\mathbf{x}=\mathbf{x}^{0}}$ the derivatives of those functions evaluated at the disease-free equilibrium (or the origin, in the case for population dynamic models). Thus, the next generation matrix is defined in terms of the two matrices:

$$
\mathcal{G}:=\mathcal{F} \mathcal{V}^{-1}
$$

Concurrently, the basic reproductive number is defined as the spectral radius (denoted by $\rho(\cdot)$ ) of the next generation matrix $\mathcal{G}$ :

$$
\mathcal{R}_{0}:=\max \{|\lambda|: \operatorname{det}(\mathcal{G}-\lambda \mathrm{id})=0\}
$$

Another formula for the spectral radius is due to Gelfand (1941), and it is given by

$$
\mathcal{R}_{0}=\lim _{m \rightarrow \infty}\left\|\mathcal{G}^{m}\right\|^{1 / m}
$$

Note that we will use the latter formulation for some inequality problems in Section 5 .

\subsection{Steps towards finding the point of interest.}

2.2.1. Implicit function theorem. The basic tool with which we will continue is the implicit function theorem. It basically concerns solvability of the implicit equation

$$
g(\mathbf{x}, \mathbf{y})=0
$$

in some neighbourhood of a point $\left(\mathbf{x}_{0}, \mathbf{y}_{0}\right)$. Details on the problem structure are given as follows.

Theorem 1. Let $\mathcal{X}, \mathcal{Y}, \mathcal{Z}$ be Banach spaces. Let $g: \mathcal{D} \rightarrow$ $\mathcal{Z}$ be some function such that $g\left(\mathbf{x}_{0}, \mathbf{y}_{0}\right)=0$ for an open set $\mathcal{D} \subset \mathcal{X} \times \mathcal{Y}$ and a tuple $\left(\mathbf{x}_{0}, \mathbf{y}_{0}\right) \in \mathcal{D}$. Additionally, suppose that $g \in C^{m}(\mathcal{D})$ for any $1 \leq m \leq \infty$, and the derivative $\partial_{\mathbf{y}} g\left(\mathbf{x}_{0}, \mathbf{y}_{0}\right)$ has a bounded inverse. Then, there exists a neighbourhood $\mathcal{U}_{\mathbf{y}}\left(\mathbf{y}_{0}\right) \subset \mathcal{Y}$ and a neighbourhood $\mathcal{U}_{\mathbf{x}}\left(\mathbf{x}_{0}\right) \subset \mathcal{X}$ such that $\mathbf{x} \in \mathcal{U}_{\mathbf{x}}\left(\mathbf{x}_{0}\right)$ admits a unique solution of (7) given by $\mathbf{y}=\Phi(\mathbf{x}) \in \mathcal{U}_{\mathbf{y}}\left(\mathbf{y}_{0}\right)$, where $\Phi$ is also of $C^{m}\left(\mathcal{U}_{\mathbf{x}}\left(\mathbf{x}_{0}\right)\right)$.

We shall clarify this with the argument that the notation $\partial_{(\cdot)}$ is used as the (Fréchet) derivative of a function, in contrast to the boundary of a set $\partial(\cdot)$. We would also use the overline notation for the closure $\overline{\mathcal{Q}}=$ $\mathcal{Q} \cup \partial \mathcal{Q}$.

The forthcoming discussion goes more into details of the process of finding a point $\left(0, \lambda_{0}\right)$ in light of the following definition.

Definition 1. (Ma and Wang, 2005) The canonical equation (2) is said to bifurcate from $\left(0, \lambda_{0}\right)$ for a fixed $\lambda_{0} \in \mathbb{R}$ a nontrivial solution $\left(\mathbf{x}_{I}, \lambda\right)$ if there exists a sequence of solutions $\left(\mathbf{x}_{I}^{(j)}, \lambda^{(j)}\right)_{j \in \mathbb{N}}$ of the equation where not all $\mathbf{x}_{I}^{(j)}$ are zero such that $\lim _{j \rightarrow \infty} \mathbf{x}_{I}^{(j)}=0$ and $\lim _{j \rightarrow \infty} \lambda^{(j)}=\lambda_{0}$. 
Clearly, direct computation of the sequence of solutions (branching solutions) would tend to answer the questions in this research, while this is considered a hard task in general. Our aim is to identify the closed form of $\lambda_{0}$ (how it is related with the canonical equation) and confirm the existence of branching solutions around $\left(0, \lambda_{0}\right)$ with the aid of topological degree theory (Krasnoselskii and Zabreiko, 1984; Ma and Wang, 2005; Nirenberg, 2001). Note that due to the nature of the canonical equation (2), we only consider degree theory for the finite dimensional case.

2.2.2. Brouwer degree. We borrow some notation and definitions from the work of Ma and Wang (2005). Let us fold the left-hand side of the canonical equation into a function $g\left(\mathbf{x}_{I}\right):=\mathbf{x}_{I}-\lambda L \mathbf{x}_{I}+\omega\left(\mathbf{x}_{I}, \lambda\right)$, where it is now independent of $\lambda$. Let $\mathcal{Q} \subseteq \mathbb{R}^{p}$ be an open set and $g: \overline{\mathcal{Q}} \rightarrow \mathbb{R}^{p}$, where $p \leq n$. In conjunction with (A1), it is easy to see that $g$ is a $C^{\infty}$ function. Let $s \in \mathbb{R}^{p}$ be a point such that $s \notin g(\partial \mathcal{Q})$. A point $\mathbf{x}_{I}^{*}$ is called regular if $\partial_{\mathbf{x}_{I}} g\left(\mathbf{x}_{I}^{*}\right)$ is invertible, otherwise it is called critical. The aforementioned point $s$ is called a regular value if either $g^{-1}(s)=\emptyset$ or all points $\mathbf{x}_{I} \in g^{-1}(s)$ are regular, otherwise it is called a critical value. The Brouwer degree of the function $g$ in an open set $\mathcal{Q}$ with respect to a point $s$, denoted by a map $\mathscr{B}: C^{1}\left(\mathcal{Q} ; \mathbb{R}^{p}\right) \times \mathcal{Q} \times \mathbb{R}^{p} \rightarrow \mathbb{Z}$, is defined as in the work of Ma and Wang (2005) by

$$
\begin{aligned}
& \mathscr{B}(g, \mathcal{Q}, s) \\
& := \begin{cases}\sum_{\mathbf{x}_{I}^{s} \in g^{-1}(s)} \operatorname{sign} \operatorname{det}\left(\partial_{\mathbf{x}_{I}} g\left(\mathbf{x}_{I}^{s}\right)\right), & s \text { regular value, } \\
\mathscr{B}\left(g, \mathcal{Q}, s^{*}\right), & s \text { critical value, } \\
& s^{*} \text { regular value },\end{cases}
\end{aligned}
$$

where, due to the latter definition, $\left\|s-s^{*}\right\|<$ $\mathrm{d}(s, g(\partial \mathcal{Q}))$. Note that $\mathrm{d}(s, A)=\inf _{a \in A}\|s-a\|$ is the standard topological distance between a point and a set. Let $\mathbf{x}_{I}^{*}$ be an isolated singular value of $g$, i.e., $g\left(\mathbf{x}_{I}^{*}\right)=0$, and suppose that the there exists $\delta>0$ such that no point in the open ball $\mathbb{B}\left(\mathbf{x}_{I}^{*} ; \delta\right)$ makes $g$ zero. The so-called index of $g$ at $\mathbf{x}_{I}^{*}$ is defined as

$$
\operatorname{ind}\left(g, \mathbf{x}_{I}^{*}\right)=\mathscr{B}\left(g, \mathcal{U}\left(\mathbf{x}_{I}^{*}\right), 0\right),
$$

where $\mathcal{U}\left(\mathbf{x}_{I}^{*}\right)$ is a neighborhood of $\mathbf{x}_{I}^{*}$. Moreover, we have previously known that $\mathbf{x}_{I}=0$ is a singular point of $g$ and it is indeed isolated with the aid of (A1)] Then, the following theorem is concerned with the index of $g$ at 0 . Let us first denote by $\alpha(\mu), \sigma(A)$ the algebraic multiplicity of an eigenvalue $\mu$ and the spectrum of eigenvalues of $A$, respectively.

Theorem 2. (Krasnoselskii and Zabreiko, 1984; Ma and Wang, 2005) Let us rewrite $g=\mathrm{id}-H$ while 0 is a singular value of $g$. If 1 is not an eigenvalue of $\partial_{\mathbf{x}_{I}} H$, then 0 is isolated and

$$
\operatorname{ind}(g, 0)=(-1)^{\gamma}
$$

where

$$
\gamma=\sum_{i: \mu_{i} \in \sigma\left(\partial_{\mathbf{x}_{I}} H(0)\right) \cap(0,1)} \alpha\left(\mu_{i}\right) .
$$

If the index of $g$ changes in the value for which $\lambda$ changes around a $\lambda_{0}$, then $\left(0, \lambda_{0}\right)$ is a point from which the canonical equation (2) bifurcates a nontrivial solution (cf. Ma and Wang, 2005). In what follows we will discover the closed form of $\lambda_{0}$ by investigating the index of a function $h$ that has a lower dimension than $g$, for which finding nontrivial solutions of $h=0$ is equivalent to finding those of the canonical equation $g=0$.

2.2.3. Lyapunov-Schmidt reduction. In an adaptation to Theorem 2 we have to first impose the following assumptions, where the further result will also settle the explanation:

(B1) The inverse $\lambda_{0}^{-1}$ is an eigenvalue of the matrix $L$ of the canonical equation.

(B2) The algebraic multiplicity of $\lambda_{0}^{-1}$ is odd.

(B3) $\lambda^{-1}$ is not an eigenvalue of $L$.

Here we will see that $\left(0, \lambda_{0}\right)$ is a such a point in Definition 1 with the aid of the Lyapunov-Schmidt reduction technique (Golubitsky and Schaeffer, 1985; Rabinowitz, 1977). Let us decompose $\mathbb{R}^{p}=Y \oplus Y^{\perp}$, where $Y:=\operatorname{ker}\left(\mathrm{id}-\lambda_{0} L\right)$ and therefore $\operatorname{dim}(Y)=$ $\alpha\left(\lambda_{0}^{-1}\right)<p, Y^{\perp}$ denotes the orthogonal complement of $Y$ in $\mathbb{R}^{p}$. Now any $\mathbf{x}_{I} \in \mathbb{R}^{p}$ can be represented as $\mathbf{x}_{I}=\mathbf{y}_{I}+\mathbf{y}_{I}^{\prime}$, where $\mathbf{y}_{I} \in Y$ and $\mathbf{y}_{I}^{\prime} \in Y^{\perp}$. Let us introduce two canonical projections $P: \mathbb{R}^{p} \rightarrow Y$ and $P^{\perp}:=\mathrm{id}-P: \mathbb{R}^{p} \rightarrow Y^{\perp}$. Observe that for any $\mathbf{y}_{I} \in Y$, $L \mathbf{y}_{I}=\lambda_{0}^{-1} \mathbf{y}_{I}$. This is evidence that we can split the canonical equation (2) into two parts:

$$
\begin{aligned}
\left(\mathrm{id}-\lambda \lambda_{0}^{-1}\right) \mathbf{y}_{I}+P \omega\left(\mathbf{y}_{I}+\mathbf{y}_{I}^{\prime}, \lambda\right) & =0 \\
(\mathrm{id}-\lambda L) \mathbf{y}_{I}^{\prime}+P^{\perp} \omega\left(\mathbf{y}_{I}+\mathbf{y}_{I}^{\prime}, \lambda\right) & =0
\end{aligned}
$$

with (9) being $\alpha\left(\lambda_{0}^{-1}\right)$-dimensional. Furthermore, the left-hand side of (10) is clearly differentiable near $\left(\mathbf{y}_{I}, \mathbf{y}_{I}^{\prime}, \lambda\right)=\left(0,0, \lambda_{0}\right)$ and equals 0 at that point. As far as (B3) is concerned, the derivative of the left-hand side of (10) with respect to $\mathbf{y}_{I}^{\prime}$ evaluated at $(0,0, \lambda)$ for $\lambda$ near $\lambda_{0}$ is just the isomorphism id $-\lambda L$, and therefore it has a bounded inverse. By the implicit function theorem, there exists a neighborhood $\mathcal{U}\left(0, \lambda_{0}\right) \subset Y \times \mathbb{R}$ and a $C^{\infty}$ function $\Phi$ defined there such that $\mathbf{y}_{I}^{\prime}=\Phi\left(\mathbf{y}_{I}, \lambda\right)$. Now, still by Eqn. (10), we know that $\Phi$ solves

$$
\Phi\left(\mathbf{y}_{I}, \lambda\right)=-(\mathrm{id}-\lambda L)^{-1} P^{\perp} \omega\left(\mathbf{y}_{I}+\Phi\left(\mathbf{y}_{I}, \lambda\right), \lambda\right)
$$


with $\omega$ enjoying (A1), which implies $\|\Phi\|=\mathcal{O}\left(\left\|\mathbf{y}_{I}\right\|^{2}\right)$ as $\mathbf{y}_{I} \rightarrow 0$ uniformly for all $\lambda$ near $\lambda_{0}$. Consequently, the eigenvalue problem (2) is now equivalent to finding $\mathbf{y}_{I}$ in $\mathcal{U}(0) \subset Y$ originated from 9 :

$$
\begin{aligned}
h\left(\mathbf{y}_{I}, \lambda\right) & :=\left(\mathrm{id}-\lambda \lambda_{0}^{-1}\right) \mathbf{y}_{I}+P \omega\left(\mathbf{y}_{I}+\Phi\left(\mathbf{y}_{I}, \lambda\right), \lambda\right) \\
& =0,
\end{aligned}
$$

where $\lambda$ is near $\lambda_{0}$ (see Rabinowitz, 1977). By the topological index theorem (Theorem 2) on the basis of (B2) and (B3), we immediately obtain

$$
\operatorname{ind}(h, 0)= \begin{cases}(-1)^{0}=1, & \lambda>\lambda_{0}, \\ (-1)^{\alpha\left(\lambda_{0}^{-1}\right)}=-1, & \lambda<\lambda_{0} .\end{cases}
$$

This shows that (11) bifurcates from $\left(0, \lambda_{0}\right)$ a nontrivial solution. This conclusion also holds for the canonical equation (2) by the aforementioned equivalence.

We will further discuss the behavior of branching nontrivial solutions near $\left(0, \lambda_{0}\right)$ and show how certain conditions can lead some of them to have positive magnitude. In conjunction with the conditions (B1) (B3), we will investigate the case where we replace $\lambda_{0}^{-1}$ with the basic reproductive number $\mathcal{R}_{0}$. We noted that the oddness of the algebraic multiplicity of $\mathcal{R}_{0}$ as required in (B2) is hard to achieve unless not only is $\mathcal{R}_{0}$ an eigenvalue of $L$, but $\mathcal{R}_{0}$ has also to be the largest eigenvalue of $L$. Then, the oddness can simply be replaced by its simplicity.

\section{Simplicity of the basic reproductive number}

Let (1) be any model that is amenable to the next generation method and has a canonical equation (2) such that the following conditions hold:

(C1) $\mathcal{R}_{0}$ is the largest eigenvalue of the matrix $L$.

(C2) $L$ is nonnegative and bounded, in the sense that each element of $L$ never has a pole in the set where all the parameters are defined.

(C3) $\lambda^{-1}$ is not an eigenvalue of $L$.

The only goal in this section is to prove the following theorem.

Theorem 3. Suppose the conditions (C1) (C2) hold. Then, the basic reproductive number $\mathcal{R}_{0}$ is simple and associated with a nonnegative eigenvector of $L$.

We will organize the proof based on the fact that the nonnegative matrix $L$ can be to which a sequence of positive matrices having simple spectral radii converges. Before doing so, we shall include several supporting lemmas.
Lemma 1. (Continuity of polynomial roots) (Ortega, 1932) Let $\mathbf{p}_{b}^{n}(\lambda):=\lambda^{n}+\sum_{j=0}^{n-1} b_{n-j} \lambda^{j}$ be a polynomial of degree $n$ having $s$ distinct roots $\lambda_{1}, \ldots, \lambda_{s}$ $(1 \leq s \leq n)$ with the corresponding algebraic multiplicities $\alpha_{1}, \ldots, \alpha_{s}$. Then, for any $\epsilon>0$ such that all disks $\overline{\mathbb{D}\left(\lambda_{1} ; \epsilon\right)}, \ldots, \overline{\mathbb{D}\left(\lambda_{s} ; \epsilon\right)}$ are disjoint, there exists $\delta=\delta(\epsilon)>0$ such that the polynomial $\mathbf{p}_{a}^{n}(\lambda):=\lambda^{n}+$ $\sum_{j=0}^{n-1} a_{n-j} \lambda^{j}$, where $\left|a_{n-j}-b_{n-j}\right|<\delta$ for all $j$, has $a$ root $\tilde{\lambda}_{i} \in \mathbb{D}\left(\lambda_{i} ; \epsilon\right)$ with algebraic multiplicity exactly $\alpha_{i}$, for all $i=1, \ldots, s$.

It might be simpler to use the notation $\mathbf{p}_{b}^{n}(\lambda)$ and $\mathbf{p}_{a}^{n}(\lambda)$ for the polynomials, where $n$ and $b$ can flexibly change. Recall the matrix $L$ in the canonical equation. We define

$$
L\left(\mu_{k}\right):=L+\mu_{k} J,
$$

where $J_{i j}=1$ if $L_{i j}=0$ and $J_{i j}=0$ if $L_{i j}>0$, $\left(\mu_{k}\right)_{k \in \mathbb{N}} \subset(0, \infty)$ a monotonically decreasing sequence converging to 0 . A glimpse over the structure of $J$ confirms that $J$ is bounded. We then get the following result.

Lemma 2. Let $A_{1}^{l}, \ldots, A_{m}^{l}$ be $p \times p$ real matrices such that $A_{i}^{l} \in\left\{L, \mu_{k} J\right\}$ where l extends from 1 to $2^{m}$ and $1 \leq m \leq p$. Suppose that $l=2^{m}$ admits $A_{1}^{l}=\cdots=$ $A_{m}^{l}=L$. Let us write the characteristic polynomial of $L$ and $L\left(\mu_{k}\right)$ as $\mathbf{p}_{a}^{p}(\lambda)$ and $\lambda^{p}+\sum_{j=0}^{p-1} b_{p-j}^{k} \lambda^{j}:=\mathbf{p}_{b^{k}}^{p}(\lambda)$, respectively. Then, the following assertions hold true:

(i) All unique combinations $A_{1}^{l} A_{2}^{l} \cdots A_{m}^{l}$ for all $l<$ $2^{m}$ have trace less than some positive definite function of $m$ multiplied by $\mu_{k}$ for all $k \geq \min \{k$ : $\left.\mu_{k}<1\right\}$.

(ii) $\left|\operatorname{Tr}\left(L\left(\mu_{k}\right)^{m}\right)-\operatorname{Tr}\left(L^{m}\right)\right|$ is bounded above by some positive definite function of $m$ multiplied by $\mu_{k}$ for all $k \geq \min \left\{k: \mu_{k}<1\right\}$.

(iii) $\left|b_{p-j}^{k}-a_{p-j}\right|$ are also bounded by some positive definite function of $j$ multiplied by $\mu_{k}$, for all $j \in$ $\{0, \ldots, p-1\}$ and for all $k \geq \min \left\{k: \mu_{k}<1\right\}$.

Proof.

Part $(i)$. Let $F$ be the Jordan normal form of $A_{1}^{l} A_{2}^{l} \cdots A_{m}^{l}$ such that there exists a nonsingular $P$ from which

$$
\begin{aligned}
\operatorname{Tr}\left(A_{1}^{l} A_{2}^{l} \cdots A_{m}^{l}\right) & =\operatorname{Tr}\left(P F P^{-1}\right)=\operatorname{Tr}\left(P^{-1} P F\right) \\
& =\operatorname{Tr}\left(F P^{-1} P\right)=\operatorname{Tr}(F) .
\end{aligned}
$$

This gives

$$
\begin{aligned}
& \operatorname{Tr}\left(A_{1}^{l} A_{2}^{l} \cdots A_{m}^{l}\right) \\
& =\sum_{\lambda \in \sigma\left(A_{1}^{l} A_{2}^{l} \cdots A_{m}^{l}\right)}^{\lambda} \\
& \leq p \rho\left(A_{1}^{l} A_{2}^{l} \cdots A_{m}^{l}\right) \leq p\left\|A_{1}^{l} A_{2}^{l} \cdots A_{m}^{l}\right\|_{1}
\end{aligned}
$$




$$
\leq p \prod_{j=1}^{m}\left\|A_{j}^{l}\right\|_{1}
$$

for all $l<2^{m}$. Now write $k^{*}:=\min \left\{k: \mu_{k}<1\right\}$. Since $A_{1}^{l} A_{2}^{l} \cdots A_{m}^{l}$ contains $\mu_{k} J$ of order at least 1 , then, together with boundedness of $\|L\|_{1}$ and $\|J\|_{1}$, there exists a bounded positive definite function $c_{l}(m)$ such that

$$
\operatorname{Tr}\left(A_{1}^{l} A_{2}^{l} \cdots A_{m}^{l}\right) \leq \mu_{k} c_{l}(m), \quad k \geq k^{*} .
$$

Part (ii). From 3 , it holds for all $1 \leq m \leq p$ that

$$
\begin{aligned}
& \left|\operatorname{Tr}\left(L\left(\mu_{k}\right)^{m}\right)-\operatorname{Tr}\left(L^{m}\right)\right| \\
& \leq\left|\sum_{l=1}^{2^{m}-1} \operatorname{Tr}\left(A_{1}^{l} \cdots A_{m}^{l}\right)\right|+\left|\operatorname{Tr}\left(L^{m}\right)-\operatorname{Tr}\left(L^{m}\right)\right| \\
& \leq \mu_{k} \sum_{l=1}^{2^{m}-1} c_{l}(m):=\mu_{k} c(m), \quad k \geq k^{*},
\end{aligned}
$$

where $c(m)$ is clearly bounded positive definite.

Part (iii). Using Fadeeva's formula as in, e.g., the work of Zadeh and Desoer (1963), one can determine the coefficients $a_{1}, \cdots, a_{p}$ (the same way as in $b_{1}^{k}, \cdots, b_{p}^{k}$ ) by the following recursive formula:

$$
\begin{gathered}
a_{1}=-\operatorname{Tr}(L), \quad a_{2}=-\frac{1}{2}\left(\operatorname{Tr}\left(L^{2}\right)+a_{1} \operatorname{Tr}(L)\right), \\
a_{j}=-\frac{1}{j}\left(\operatorname{Tr}\left(L^{j}\right)+a_{1} \operatorname{Tr}\left(L^{j-1}\right)+\cdots\right. \\
\left.\quad+a_{j-1} \operatorname{Tr}(L)\right), \quad 3 \leq j \leq p .
\end{gathered}
$$

Note that one must replace $L$ with $L\left(\mu_{k}\right)$ in the last formula in order to compute $b_{1}^{k}, \ldots, b_{p}^{k}$. We shall reveal the fact that $\operatorname{Tr}\left(L^{m}\right) \leq p\left\|L^{m}\right\|_{1} \leq p\|L\|_{1}^{m}<\infty$ for finite $p$ and $\operatorname{Tr}\left(L\left(\mu_{k}\right)^{m}\right) \leq p\left\|L+\mu_{k} J\right\|_{1}^{m} \leq$ $p \max _{i \in\{0, \ldots, m\}}\left(\begin{array}{c}m \\ i\end{array}\right)\|L\|_{1}^{i}\left\|\mu_{k} J\right\|_{1}^{m-i}<\infty$ for all $1 \leq$ $m \leq p$ and finite $p$. We now conclude that $\left|a_{j}\right|,\left|b_{j}^{k}\right|$ are bounded for all finite $j$. Then we obtain for all $k \geq k^{*}$,

$$
\begin{aligned}
\left|b_{1}^{k}-a_{1}\right|= & \left|\operatorname{Tr}\left(L\left(\mu_{k}\right)\right)-\operatorname{Tr}(L)\right| \\
\leq & \mu_{k} c(1)=: \mu_{k} d(1), \\
\left|b_{2}^{k}-a_{2}\right| \leq & \frac{1}{2}\left\{\left|\operatorname{Tr}\left(L\left(\mu_{k}\right)^{2}\right)-\operatorname{Tr}\left(L^{2}\right)\right|\right. \\
& +\left|a_{1}\right| \mid \operatorname{Tr}\left(L\left(\mu_{k}\right)\right) \\
& \left.-\operatorname{Tr}(L)|+| b_{1}^{k}-a_{1}|| \operatorname{Tr}\left(L\left(\mu_{k}\right)\right) \mid\right\} \\
\leq & \frac{1}{2}\left\{\mu_{k} c(2)+\left|a_{1}\right| \mu_{k} d(1)\right. \\
& \left.+\left|\operatorname{Tr}\left(L\left(\mu_{k}\right)\right)\right| \mu_{k} d(1)\right\}=: \mu_{k} d(2), \\
\left|b_{j}^{k}-a_{j}\right| \leq & \frac{1}{j}\left\{\left|\operatorname{Tr}\left(L\left(\mu_{k}\right)^{j}\right)-\operatorname{Tr}\left(L^{j}\right)\right|\right. \\
& +\sum_{l=1}^{j-1}\left|a_{l}\right| \mid \operatorname{Tr}\left(L\left(\mu_{k}\right)^{l}\right)
\end{aligned}
$$

$$
\begin{aligned}
& \left.-\operatorname{Tr}\left(L^{l}\right)\left|+\sum_{l=1}^{j-1}\right| b_{l}^{k}-a_{l}|| \operatorname{Tr}\left(L\left(\mu_{k}\right)^{l}\right) \mid\right\} \\
\leq & \frac{1}{j}\left\{\mu_{k} c(j)+\sum_{l=1}^{j-1}\left|a_{l}\right| \mu_{k} c_{l}\right. \\
& \left.+\left|\operatorname{Tr}\left(L\left(\mu_{k}\right)^{l}\right)\right| \mu_{k} d(l)\right\} \\
= & : \mu_{k} d(j), \quad 3 \leq j \leq p,
\end{aligned}
$$

which completes the proof.

Corollary 1. $\left|b_{p-j}^{k}-b_{p-j}^{r}\right|$ are bounded by some positive definite function of $j$ multiplied by $\left|\mu_{k}-\mu_{q}\right|$, for $0 \leq j \leq$ $p-1$ and for some large $k, r$.

Now we have all what we need to prove Theorem 3

Proof. (Theorem 3) We shall divide the proof into two main parts: (i) that $\mathcal{R}_{0}$ is simple and (ii) that $\mathcal{R}_{0}$ associates with a nonnegative eigenvector. The first part follows directly from Lemmas 2$]$ and 1 . Suppose that $\lambda_{1}(k), \ldots, \lambda_{s}(k)(1 \leq s \leq p)$ are the eigenvalues of $L\left(\mu_{k}\right)$ with the corresponding algebraic multiplicities $\alpha_{1}, \ldots, \alpha_{s}$, i.e., they are the roots of $\mathbf{p}_{b^{k}}^{p}(\lambda)$. Now choose $\epsilon>0$ small enough such that all disks $\overline{\mathbb{D}\left(\lambda_{1}(k) ; \epsilon\right)}, \ldots, \overline{\mathbb{D}\left(\lambda_{s}(k) ; \epsilon\right)}$ are disjoint. Then, there exists $\delta>0$ such that $\left|a_{p-j}-b_{p-j}^{k}\right|<\delta$ for all $j \in$ $\{0, \ldots, p-1\}$, where $a_{1}, \ldots, a_{p}$ are the coefficients of the polynomial $\mathbf{p}_{a}^{p}(\lambda)$.

We can define

$$
k^{* *}:=\min \left\{k \geq k^{*}: \mu_{k} \max _{j \in\{1, \ldots, p\}} d(j)<\delta\right\}
$$

for $d(j), k^{*}$ given as in Lemma 2, This means $\mid a_{p-j}-$ $b_{p-j}^{k} \mid \leq \mu_{k} \max _{j \in\{1, \ldots, p\}} d(j)<\delta$ for all $k \geq k^{* *}$, and therefore $\mathbf{p}_{a}^{p}(\lambda)$ is the characteristic polynomial of $L$. Furthermore, the Perron-Frobenius theorem guarantees that $\rho\left(L\left(\mu_{k}\right)\right)$ is simple for all $1 \leq k<\infty$, and so is $\mathcal{R}_{0}=\rho(L)$. This shows that we can choose a decreasing sequence of positive numbers $\left(\epsilon_{k}\right)_{k \geq K}$ that converges to 0 for $K$ sufficiently large, so that there is a sequence of positive numbers $\left(\delta_{k}\right)_{k \geq K}$. In return, we can point out a sequence of positive numbers $\left(c_{k}\right)_{k \geq K}$ where $c_{k}<\infty$ such that $\epsilon_{k} \leq c_{k} \mu_{k}$ for all $K \leq k<\infty$.

For the second part, it follows from the Perron-Frobenius theorem that not only is $\rho\left(L\left(\mu_{k}\right)\right)$ simple, but it also associates with a positive eigenvector $v\left(\mu_{k}\right)$, where $\left\|v\left(\mu_{k}\right)\right\|=1$ for all $k<\infty$. Let us take $k \geq K$. Let $v$ be a normal vector, i.e., $\|v\|=1$, such that $\left(L-\mathcal{R}_{0} \mathrm{id}\right) v=0$. Let us define some projection $P:=\left\langle v\left(\mu_{k}\right), v\right\rangle v$ and therefore $P^{\perp}:=v\left(\mu_{k}\right)-P$ such that $\left\langle P, P^{\perp}\right\rangle=$ $\left\langle P, v\left(\mu_{k}\right)\right\rangle-\|P\|^{2}=\left\langle v\left(\mu_{k}\right), v\right\rangle^{2}-\left\langle v\left(\mu_{k}\right), v\right\rangle^{2}\|v\|^{2}=0$ and $\left\|P+P^{\perp}\right\|=\left\|v\left(\mu_{k}\right)\right\|=1$. Our aim is to prove that there exists a sequence of bounded positive numbers $\left(d_{k}\right)_{k \geq K}$ such that $\left\|P^{\perp}\right\| \leq d_{k} \mu_{k}$ towards 
the following destination. If this premise holds, then by means of $\left\|P+P^{\perp}\right\|=\left\|v\left(\mu_{k}\right)\right\|=1$, we gain $0 \leq 1-\left|\left\langle v\left(\mu_{k}\right), v\right\rangle\right|=1-\|P\| \leq\left\|P^{\perp}\right\| \leq d_{k} \mu_{k}$. Consequently, as $k \rightarrow \infty$, we have $v\left(\mu_{k}\right) \rightarrow v$ due to the squeeze theorem.

To show that $\left\|P^{\perp}\right\| \leq d_{k} \mu_{k}$, we first assume that $v\left(\mu_{k}\right) \neq v$ since otherwise everything boils down to triviality. Let us introduce an auxiliary matrix $B\left(\mu_{k}\right):=$ $L\left(\mu_{k}\right)-\rho\left(L\left(\mu_{k}\right)\right)$ id such that $B\left(\mu_{k}\right) v\left(\mu_{k}\right)=0$, and for simplicity $B:=B(0)$. In light of the distinction between $v\left(\mu_{k}\right)$ and $v$, we immediately confirm that $B v\left(\mu_{k}\right) \neq 0$, or there exists $\varepsilon_{0}>0$ such that $\left\|B v\left(\mu_{k}\right)\right\|=\varepsilon_{0}$. At the expense of making a contradiction, let us first suppose that $\left\|B P^{\perp}\right\|<\varepsilon_{1}\left\|P^{\perp}\right\|$ for all $\varepsilon_{1}>0$. This is equivalent to stating that $\left\|B v\left(\mu_{k}\right)\right\|<\varepsilon_{1}\left\|v\left(\mu_{k}\right)-\left\langle v\left(\mu_{k}\right), v\right\rangle v\right\| \leq$ $2 \varepsilon_{1}:=\varepsilon$ for all $\varepsilon>0$, which is no longer valid for any $\varepsilon \leq \varepsilon_{0}$. Eventually, there must exist a small $\varepsilon>0$ such that $\left\|B P^{\perp}\right\| \geq \varepsilon\left\|P^{\perp}\right\|$. Together with

$$
\begin{aligned}
B P^{\perp} & =B v\left(\mu_{k}\right)-B P=B v\left(\mu_{k}\right)-\underbrace{\left\langle v\left(\mu_{k}\right), v\right\rangle B v}_{=0=B\left(\mu_{k}\right) v\left(\mu_{k}\right)} \\
& =\left(B-B\left(\mu_{k}\right)\right) v\left(\mu_{k}\right),
\end{aligned}
$$

these facts reveal that

$$
\begin{aligned}
& \left\|P^{\perp}\right\| \\
& \leq \frac{\left\|\left(B-B\left(\mu_{k}\right)\right) v\left(\mu_{k}\right)\right\|}{\varepsilon} \\
& \leq \frac{\left|\mathcal{R}_{0}-\rho\left(L\left(\mu_{k}\right)\right)\right|+\|J\| \mu_{k}}{\varepsilon} \leq \underbrace{\left(\frac{c_{k}}{\varepsilon}+\frac{\|J\|}{\varepsilon}\right)}_{:=d_{k}} \mu_{k}
\end{aligned}
$$

for all $k \geq K$. At this stage we have shown that $v\left(\mu_{k}\right) \rightarrow$ $v$. Continuity of the root in the first part, together with the fact that all $v\left(\mu_{k}\right)$ are positive for all $k<\infty$, makes an argument that $v$ is therefore nonnegative.

We can also check by irreducibility that if a nonnegative matrix $L$ has $\mathcal{R}_{0}$ as the simple largest eigenvalue, then $\mathcal{R}_{0}$ associates with a positive eigenvector. A nonnegative matrix $L$ is said to be reducible if it is permutationally similar to a block upper triangular matrix and irreducible if it is not reducible. To some extent, it is considered easier to see if a nonnegative matrix is irreducible by investigating its corresponding directed graph. To any nonnegative matrix $L=\left(l_{i j}\right)_{i, j=1, \ldots, p}$ we can derive a directed graph $D(L)$ containing a set of $p$ vertices $v_{1}, \ldots, v_{p}$ and a set of directed edges $v_{i} \rightarrow v_{j}$ $\left(i \neq j\right.$ ) whose cost to go from $v_{i}$ to $v_{j}$ is represented by $l_{i j}$. If $l_{i j}=0$, we can say there is no directed edge from $v_{i}$ to $v_{j}$. What we then refer to as a path is a collection of one or more directed edges; for example, we may call $v_{i} \rightarrow v_{j} \rightarrow v_{k}$ a path from $v_{i}$ to $v_{k}$. The directed graph of a nonnegative matrix $L$ is said to be strongly connected if for any vertices $v_{i}, v_{j}$ in $D(L)$ there exists a path from $v_{i}$ to $v_{j}$. In other words, a strongly connected directed graph possesses vertices that can be accessed from any other vertices. The following lemma gives, instead of permutation, another way to check if a matrix is irreducible.

Lemma 3. A nonnegative matrix $L$ is irreducible if and only if the corresponding directed graph $D(L)$ is strongly connected.

Following this lemma is an important complement to Theorem 3 that opens a way to see the positivity of the eigenvector that associates with $\mathcal{R}_{0}$.

Theorem 4. (Perron-Frobenius theorem for irreducible nonnegative matrices) If a nonnegative matrix $L$ is irreducible, then $\rho(L)$ is a simple eigenvalue of $L$ and associates with a positive eigenvector called $a$ Perron vector.

Note that the last theorem only gives a sufficient condition. If the irreducibility test fails, then this does not always mean that the spectral radius cannot associate with a positive eigenvector.

\section{Notes on global bifurcation}

Let us stick to the conditions (C1) (C3). The following theorem is well known as the Rabinowitz global bifurcation theorem. It basically amounts to reviewing the properties of branching solutions near the point $\left(0, \mathcal{R}_{0}^{-1}\right)$ in more detail.

Theorem 5. (Rabinowitz, 1971) Denote by

$$
\Sigma_{0}:=\left\{\left(\mathbf{x}_{I}, \lambda\right) \in \mathbb{R}^{p} \times \mathbb{R}: \mathbf{x}_{I} \neq 0,\left(\mathbf{x}_{I}, \lambda\right) \text { solves (2) }\right\}
$$

the set of nontrivial solutions to (2) and by $\Sigma$ a bounded open subset of $\mathbb{R}^{p} \times \mathbb{R}$ containing $\left(0, \mathcal{R}_{0}^{-1}\right)$. Let $\omega$ be continuous and compact on $\Sigma$ and $\mathcal{R}_{0}$ be the largest eigenvalue of $L$; then there exists a continuum of solutions $\mathcal{C} \subset \Sigma_{0}$ where $\left(0, \mathcal{R}_{0}^{-1}\right) \in \mathcal{C}$, and either (i) $\mathcal{C}$ meets $\partial \Sigma$ or (ii) $\mathcal{C}$ meets $\left(0, \mathcal{R}^{-1}\right)$, where $\mathcal{R}_{0} \neq \mathcal{R}$ is another eigenvalue of $L$ with odd algebraic multiplicity.

To adapt to the theorem, we have designated

$$
\Sigma:=\mathcal{Q}_{0} \times(-1,1),
$$

where $\mathcal{Q}_{0} \subset \mathbb{R}^{p}$ is a bounded open subset containing 0 . Choosing

$$
\mathcal{R}_{0}>1
$$

immediately confirms $\left(0, \mathcal{R}_{0}^{-1}\right) \in \Sigma$. As for Theorem 5 , there exists a continuum of solutions $\mathcal{C}$ containing the trivial solution $\left(0, \mathcal{R}_{0}^{-1}\right)$ that meets either $\partial \Sigma$ or another trivial solution $\left(0, \mathcal{R}^{-1}\right)$, where $\mathcal{R}$ is another eigenvalue of $L$ with odd algebraic multiplicity. The forthcoming remarks identify the closed form of the nontrivial solutions in $\mathcal{C}$, which is

$$
\mathbf{x}_{I}(t)=t v+u(t), \quad \lambda(t)=\mathcal{R}_{0}^{-1}-\chi(t),
$$


where $v$ is the right eigenvector of $L$ associated with $\mathcal{R}_{0}^{-1}$, $t \in \mathbb{R} \backslash\{0\}$ and $\lim _{t \rightarrow 0^{ \pm}}\left(\mathbf{x}_{I}(t), \chi(t)\right)=(0,0)$. In the case of $u$ being $\mathcal{O}\left(t^{2}\right)$ as $t \rightarrow 0$, the initial direction of the solution $\mathbf{x}_{I}$ on the chosen domain $(0, \infty)$ is determined by $\left.\partial_{t} \mathbf{x}_{I}\right|_{t=0}=v$.

To proceed, consider the following rearrangement of the canonical equation:

$$
0=\left(\mathrm{id}-\mathcal{R}_{0}^{-1} L\right) \mathbf{x}_{I}+\left(\mathcal{R}_{0}^{-1}-\lambda\right) L \mathbf{x}_{I}+\omega\left(\mathbf{x}_{I}, \lambda\right) .
$$

Let $w$ and $v$ be respectively the left and right eigenvectors of $\mathcal{R}_{0}^{-1} L$ associated with the eigenvalue 1 . For any $\mathbf{x}_{I} \in \mathbb{R}^{p}$ there exists $t \in \mathbb{R}$ and $u \in \operatorname{span}\{v\}^{\perp}$ such that $\mathbf{x}_{I}=t v+u$, as desired. The next crucial step is checking the order of $u$ with respect to $t$. From (15) we obtain $w^{\prime}\left(\left(\mathcal{R}_{0}^{-1}-\lambda\right) L \mathbf{x}_{I}+\omega\left(\mathbf{x}_{I}, \lambda\right)\right)=-w^{\prime}\left(\mathrm{id}-\mathcal{R}_{0}^{-1} L\right) \mathbf{x}_{I}=0$, confirming $\left(\mathcal{R}_{0}^{-1}-\lambda\right) L \mathbf{x}_{I}+\omega\left(\mathbf{x}_{I}, \lambda\right) \in \operatorname{span}\{w\}^{\perp}$. This tells us that for each defined $\mathbf{x}_{I} \in \mathbb{R}^{p}$, making $\left(\mathcal{R}_{0}^{-1}-\lambda\right) L \mathbf{x}_{I}+\omega\left(\mathbf{x}_{I}, \lambda\right) \in \operatorname{span}\{w\}^{\perp}$, there exists a point $u \in \operatorname{span}\{v\}^{\perp}$ such that $\mathbf{x}_{I}=t v+u$. This exposition leads to the existence of a linear transformation $\Delta: \operatorname{span}\{w\}^{\perp} \rightarrow \operatorname{span}\{v\}^{\perp}$ mapping $\left(\mathcal{R}_{0}^{-1}-\lambda\right) L \mathbf{x}_{I}+$ $\omega\left(\mathbf{x}_{I}, \lambda\right)$ into $u$ (cf. Cushing, 1998). Decomposing $\mathbf{x}_{I}$, the very last assertion means

$$
\begin{aligned}
u= & \left(\mathcal{R}_{0}^{-1}-\lambda\right) L t \Delta v \\
& +\left(\mathcal{R}_{0}^{-1}-\lambda\right) L \Delta u+\Delta \omega(t v+u, \lambda) .
\end{aligned}
$$

Let $\chi:=\mathcal{R}_{0}^{-1}-\lambda$, which is deduced to be $t$-dependent. Clearly, the function $\Theta$ in $\Theta(u, t, \chi)=0$ as a short-hand writing of (16) is infinitely differentiable by (A1) and the derivative is given by $\partial_{u} \Theta(0,0,0)=\mathrm{id}$. By the implicit function theorem, there exists a neighborhood $\mathcal{U}(0,0) \subset \mathbb{R} \times \mathbb{R}$ and a $C^{\infty}$ function $u=u(t, \chi)$ defined there solving (16). Equation (16) also tells us that $u(0, \chi)=0$ for $\chi$ in that neighborhood. Furthermore, taking the derivative of (16) with respect to $t$ results in

$$
\begin{aligned}
\partial_{t} u= & \partial_{t} \chi L t \Delta v+\chi L \Delta v+\partial_{t} \chi L \Delta u+\chi L \partial_{u} \Delta u \partial_{t} u \\
& +\partial_{\omega} \Delta \omega\left(\partial_{t} \omega+\partial_{u} \omega \partial_{t} u-\partial_{\lambda} \omega \partial_{t} \chi\right),
\end{aligned}
$$

where we obtain the specification $\partial_{t} u(0,0)=0$ by (A1). Taking the first derivative of (16) with respect to $\chi$, we have

$$
\partial_{\chi} u=L t \Delta v+L \Delta u+\partial_{\omega} \Delta \omega\left(\partial_{u} \omega \partial_{\chi} u-\partial_{\lambda} \omega\right)
$$

such that $\partial_{\chi} u(0,0)=0$. The Taylor expansion $u(t, \chi)=$ $u(0,0)+\partial_{t} u(0,0) t+\partial_{\chi} u(0,0) \chi+\mathcal{O}\left(\|(t, \chi)\|^{2}\right)$ suggests that $u=\mathcal{O}\left(t^{2}\right)$ uniformly for $\chi$ near 0 , as desired. Moreover, we know that $w^{\prime}\left(\chi L \mathbf{x}_{I}+\omega\right)=w^{\prime}(\chi L t v+$ $\chi L u+\omega)=0$ with $\omega$ enjoying (A1) By the implicit function theorem, there exists a neighborhood $\mathcal{U}(0) \subset \mathbb{R}$ and a $\chi=\chi(t)$ of $C^{\infty}$ solving the last equation for $t \in \mathcal{U}(0)$, satisfying $\chi(0)=0$. This gives us extra information concerning the desired property of $\chi$.

Summarizing what we have done so far, we get our main result.
Theorem 6. Let (1) be any model that is amenable to the next generation method and has a canonical equation (2) where (C1) (C3) hold. Then the basic reproductive number $\mathcal{R}_{0}$ is simple and associates with a nonnegative eigenvector $v \neq 0$ of $L$. If $\mathcal{R}_{0}>1$ is sufficiently near $\lambda^{-1}$, then there exists a nonnegative nontrivial solution of the canonical equation whose magnitude is drifted by $v$. Furthermore, if $L$ is irreducible, then there exists a strictly positive nontrivial solution.

\section{Examples}

To see how this approach works, we consider three models $(\mathcal{M} 1)(\mathcal{M} 2)$ and $(\mathcal{M} 3)$ whose transmission diagrams are depicted as in Fig.11

$(\mathcal{M} 1)$ describes the dynamics of a mosquito population based on the age and indoor-outdoor classification (Wijaya et al., 2014). The population is divided into 5 compartments: indoor egg $E_{1}$, outdoor egg $E_{2}$, indoor larva $L_{1}$, outdoor larva $L_{2}$, and adult $A$. Every egg is supposed to transform into a larva at the rates of transition $\alpha_{1}$ (indoor) and $\alpha_{2}$ (outdoor) while a larva transforms into a pupa at the rates $\beta_{1}$ (indoor) and $\beta_{2}$ (outdoor). Under the limitation of logistics, larvae that live side by side compete for food with the competition rates $\sigma_{1}, \sigma_{2}$. We denote by $\mu$ the growth rate of eggs, where the probabilistic term $p$ distinguishes the growth rate of indoor eggs. The natural death rates are denoted by $\eta_{1}, \ldots, \eta_{5}$. For the sake of applying treatments, it is assumed that an amount of larvicide is disposed into indoor water vessels at the rate $u_{1}$ for killing larvae and partly eggs (indicated by $q$ ). Also, fumigation is performed at the rate $u_{2}$ to kill adults. We shall add the following specifications to justify some variables regarding $(\mathcal{M} 1)$ in Table $1 s_{1}:=\mu p, s_{2}:=\mu(1-p)$, $s_{3}:=\alpha_{1}, s_{4}:=\alpha_{2}, s_{5}:=\beta_{1}, s_{6}:=\beta_{2}$ and $d_{1}:=$ $\alpha_{1}+\eta_{1}+q u_{1}, d_{2}:=\alpha_{2}+\eta_{2}, d_{3}:=\beta_{1}+\eta_{3}+u_{1}$, $d_{4}:=\beta_{2}+\eta_{4}, d_{5}:=\eta_{5}+u_{2}$,

$$
\begin{aligned}
& a_{1}:=\frac{d_{3}}{\sigma_{1}}-\frac{s_{1} s_{3} s_{5}}{d_{1} d_{5} \sigma_{1}} \\
& a_{2}:=\frac{d_{4}}{\sigma_{2}}-\frac{s_{2} s_{4} s_{6}}{d_{2} d_{5} \sigma_{2}} .
\end{aligned}
$$

We assume that

$$
a_{1}>0, \quad a_{2}>0 .
$$

According to Wijaya et al. (2014, Theorem 3.3), the classical direct calculation confirms the existence of a coexistence equilibrium providing $\mathcal{R}_{0}>1$.

$(\mathcal{M} 2)$ describes a host-vector model for dengue epidemics including vaccination treatment. It captures the dynamics of host compartments: susceptible $S$, infective $I$, recovered $R$ and vaccinated $C$; and vector compartments: susceptible $U$ and infective $V$. We 


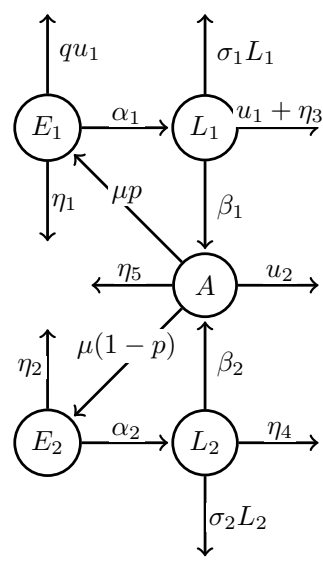

$(\mathcal{M} 1)$

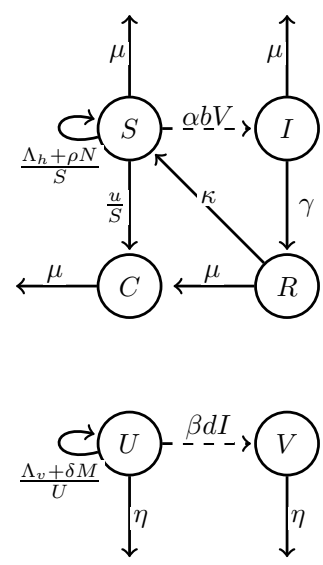

$(\mathcal{M} 2)$

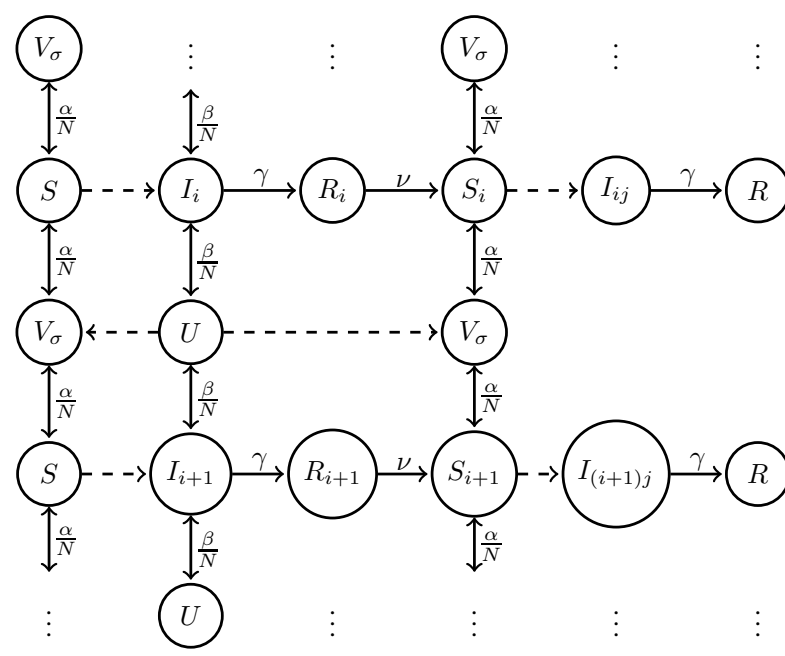

$(\mathcal{M} 3)$

Fig. 1. Three sample models. $\rightarrow, \uparrow, \leftarrow, \downarrow$ : transitions to other compartments or to deaths, $\downarrow$ : contact, $-\rightarrow, \leftarrow--$ : transitions due to contacts.

assume that each newborn is susceptible, contributed by constant recruitments at the rates $\Lambda_{h}$ (host) and $\Lambda_{v}$ (vector), respectively, and additional growth factors at the rates $\rho$ and $\delta$. A susceptible host and, respectively, a susceptible vector that are in contact with an infective vector and an infective human clinically move to the infective compartments along with the driving forces of infection whose rates are $\alpha, \beta$. The terms $b, d$ stand for the average biting rate of an infective vector to a susceptible host and that of a susceptible vector to an infective host, respectively. An infective host can experience recovery at the rate $\gamma$. Due to loss of immunity, a recovered host moves back to the susceptible compartment at the rate $\kappa$. Moreover, a constant portion $u$ of susceptible hosts is treated using vaccination from which they immediately move to the vaccinated compartment. The average natural death rates for both host and vector populations are given by $\mu$ and $\eta$, respectively. For reasons of well-posedness, we shall assume that all the parameters are positive and

$$
\mu>\rho, \quad \eta>\delta, \quad \Lambda_{h}>u .
$$

We denote by $N:=S+I+R+C$ and $M:=U+$ $V$ the size of the total host population and that of the total vector population, respectively. A computer algebra system, e.g., Maple, was able to show up an endemic equilibrium of $(\mathcal{M} 2)$. The equilibrium happens to be of a long expression for which we cannot determine its explicit relationship with $\mathcal{R}_{0}$ and omit writing here.

$(\mathcal{M} 3)$ describes a dengue epidemic model wherein $n \geq 2$ strains of dengue virus actively contribute to new infections among humans. Assume that the host population admits a constant size $N$ from time to time. This constant population is then subdivided into several compartments: susceptible $S$, infected by the $i$-th strain $I_{i}$, recovered from the $i$-th strain $R_{i}$, susceptible to other strains except the $i$-th strain $S_{i}$, infected by the $j$-th strain after the $i$-th strain $I_{i j}$, and fully recovered $R$. We assume that any host only can get infected by a maximum of two strains during the average lifetime period.

Additionally, the notion of co-infection, i.e., that a host can be infected by viruses from two or more different strains at the same time, is not admitted in the model. In the vector population, the population size $M$ is subdivided into susceptible $U$ and co-infected from all strains indicated by a set of strain indices $\sigma \in 2^{\langle n\rangle} \backslash\{0\}$, denoted by $V_{\sigma}$, where $\langle n\rangle:=\{1, \ldots, n\}$. For example, $V_{\{1,3,10\}}$ represents the number of vectors co-infected by the first strain, third, and tenth strain, respectively. We assume that any newborn in the host population is susceptible at the given birth rate $\mu$; any human dies at the rate $\mu$, too. Any vector dies at the rate $\eta$. The other parameters include the infection rates $\alpha, \beta$, the recovery rate $\gamma$, and the loss-of-immunity rate $\kappa$.

Assume that in each contact with $V_{\sigma}$, a susceptible host can get infected by all strains in $\sigma$, with the probability $p_{i}^{\sigma}$ when subjected to the $i$-th strain. Furthermore, the probability of getting infected by only the $i$-th strain, i.e., when $\sigma=\{i\}$, must be equal to 1 . Therefore, we may define 


$$
p_{i}^{\sigma}:= \begin{cases}1, & \{i\}=\sigma, \\ \in(0,1), & i \in \sigma\end{cases}
$$

and for fixed $\sigma$ we have

$$
\sum_{i \in \sigma} p_{i}^{\sigma}=1
$$

As far as the compartment $I_{i j}$ is concerned, the growth of $I_{i j}$ is supplemented by the contact between $S_{i}$ and $V_{\sigma}$, where $\sigma$ contains $j$, with the underlying probability $q_{i j}^{\sigma}$. This probabilistic term is defined as

$$
q_{i j}^{\sigma}:= \begin{cases}1, & \{j\}=\sigma \\ \in(0,1], & j \in \sigma\end{cases}
$$

and for fixed $i$ and $\sigma$

$$
\sum_{j: j \in \sigma} q_{i j}^{\sigma}=1
$$

Moreover, for the sake of simplicity, we assume that all contacts between $U$ and $I_{i}$ will give rise to $V_{\sigma}$ as long as $\sigma$ contains $i$. Along with this assumption, we shall introduce the probabilistic terms $r_{\sigma}^{i}, s_{\sigma}^{i j}$, where $r_{\sigma}^{i} \in(0,1)$ if $i \in \sigma$ and $s_{\sigma}^{i j} \in(0,1)$ if $j \in \sigma$ and $i \neq j$, satisfying

$$
\text { for fixed } i: \quad \sum_{\sigma: i \in \sigma} r_{\sigma}^{i}=1 \quad \text { and } \quad \sum_{\sigma: j \in \sigma} s_{\sigma}^{i j}=1 \text {. }
$$

In the same way as before, the contacts between $U$ and $I_{i j}$ also give rise to $V_{\sigma}$, which bring into use $s_{\sigma}^{i j}$ while taking into consideration that only viruses with the $j$-th strain indeed reside in $I_{i j}$.

In its entirety, the model $(\mathcal{M} 3)$ is given by

$$
\begin{aligned}
\dot{S} & =\mu N-\sum_{\sigma \in 2^{\langle n\rangle} \backslash \emptyset} \frac{\alpha}{N} S V_{\sigma}-\mu S, \\
\dot{I}_{i} & =\sum_{\sigma: i \in \sigma} \frac{\alpha}{N} S p_{i}^{\sigma} V_{\sigma}-(\gamma+\mu) I_{i}, \\
\dot{R}_{i} & =\gamma I_{i}-(\nu+\mu) R_{i}, \\
\dot{S}_{i} & =\nu R_{i}-\sum_{\sigma: \sigma \neq\{i\}} \frac{\alpha}{N} S_{i} V_{\sigma}-\mu S_{i}, \\
\dot{I}_{i j} & =\sum_{\sigma: j \in \sigma} \frac{\alpha}{N} S_{i} q_{i j}^{\sigma} V_{\sigma}-(\gamma+\mu) I_{i j}, \\
\dot{R} & =\gamma \sum_{i \in\langle n\rangle} \sum_{\substack{j \in\langle n\rangle \\
j \neq i}} I_{i j}-\mu R, \\
\dot{U} & =\Lambda-\sum_{i \in\langle n\rangle} \frac{\beta}{N} U\left(I_{i}+\sum_{\substack{j \in\langle n\rangle \\
j \neq i}} I_{i j}\right)-\eta U, \\
\dot{V}_{\sigma} & =\sum_{i: i \in \sigma}\left(\frac{\beta}{N} U r_{\sigma}^{i} I_{i}\right) \quad
\end{aligned}
$$

$$
+\sum_{j: j \in \sigma} \sum_{i: i \neq j}\left(\frac{\beta}{N} U s_{\sigma}^{i j} I_{i j}\right)-\eta V_{\sigma}
$$

where $i, j \in\langle n\rangle, j \neq i$ and $\sigma \in 2^{\langle n\rangle} \backslash \emptyset$.

It is immediate to unfold the two matrices $\mathcal{F}$ and $\mathcal{V}$ from the framework of the next generation method,

$$
\mathcal{F}=\left(\begin{array}{c|c}
0 & \mathcal{F}_{12} \\
\hline \mathcal{F}_{21} & 0
\end{array}\right) \text { and } \mathcal{V}=\left(\begin{array}{c|c}
\mathcal{V}_{11} & 0 \\
\hline 0 & \mathcal{V}_{22}
\end{array}\right)
$$

where

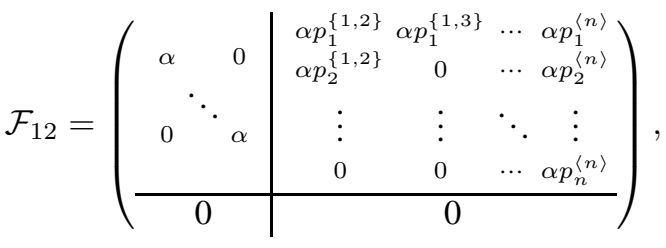

$$
\begin{aligned}
& \mathcal{F}_{21}=\left(\begin{array}{cccc|c}
\frac{\beta}{N} U^{0} r_{\{1\}}^{1} & 0 & & \left(F_{21}\right)_{12} \\
& \ddots & & & \\
0 & \frac{\beta}{N} U^{0} r_{\{n\}}^{n} & \\
\hline \frac{\beta}{N} U^{0} r_{\{1,2\}}^{1} & \frac{\beta}{N} U^{0} r_{\{1,2\}}^{2} & \cdots & 0 & \\
\frac{\beta}{N} U^{0} r_{\{1,3\}}^{1} & 0 & \cdots & 0 & \left(F_{21}\right)_{22} \\
\vdots & \vdots & \ddots & \vdots & \\
\frac{\beta}{N} U^{0} r_{\langle n\rangle}^{1} & \frac{\beta}{N} U^{0} r_{\langle n\rangle}^{2} & \cdots & \frac{\beta}{N} U^{0} r_{\langle n\rangle}^{n} &
\end{array}\right), \\
& U^{0}=\Lambda / \eta, \mathcal{V}_{11}=(\gamma+\mu) \text { id, and } \mathcal{V}_{22}=\eta \text { id. }
\end{aligned}
$$

Then, we have the next generation matrix

$$
\mathcal{G}=\left(\begin{array}{c|c}
0 & \frac{\mathcal{F}_{12}}{\eta} \\
\hline \frac{\mathcal{F}_{21}}{\gamma+\mu} & 0
\end{array}\right) .
$$

We will see that the basic reproductive number $\mathcal{R}_{0}$ is not only the spectral radius of $\mathcal{G}$ but also the largest eigenvalue of $\mathcal{G}$ and that of the matrix $L$.

Lemma 4. For the model (M3), the basic reproductive number $\mathcal{R}_{0}$ is an eigenvalue of the next generation matrix $\mathcal{G}$ and is positive ( $\mathcal{G}$ is not a nilpotent matrix).

Proof. That $\mathcal{R}_{0}$ is apparently an eigenvalue of $\mathcal{G}$ was already shown by, e.g., Horn and Johnson (2013, Theorem 8.3.1).

Now let us prove the positivity of $\mathcal{R}_{0}$. First of all, it is essential to highlight several facts regarding nonnegative matrices. If $P, Q$ are two nonnegative matrices, where $P \leq Q$ holds componentwisely, then for any induced norm $\|\cdot\|$ there exists a nonnegative vector $y$, where $\|y\|=1$ such that $\|P\|:=\|P y\| \leq$ $\|Q y\| \leq\|Q\|$. Moreover, one can immediately see that $P^{m} \leq Q^{m}$ componentwisely for any $m \geq 1$. Combining the previous expositions, we have $\left\|P^{m}\right\| \leq$ $\left\|Q^{m}\right\|$. Now we will use this result to justify the main problem. Set $r:=\min \left\{\alpha / \kappa, \beta U^{0} /(N(\gamma+\mu))\right\}$ and a matrix $\mathcal{H}=\mathcal{H}_{i j}:=r \mathcal{G}_{i j}\left(\sum_{i} \mathcal{G}_{i j}\right)^{-1}$ such that $\mathcal{H} \leq \mathcal{G}$ componentwisely and $\sum_{i} \mathcal{H}_{i j}=r$ for all $j$. 


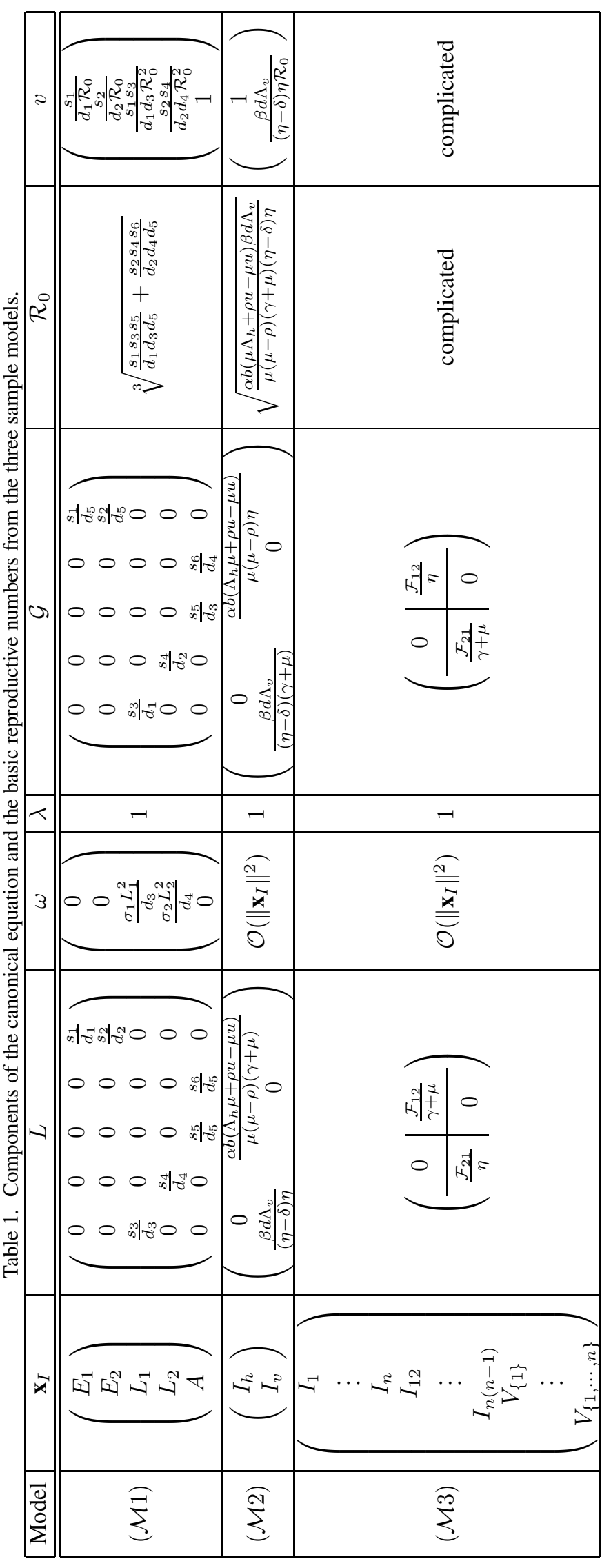

Moreover, $(1, \ldots, 1)^{\prime}$ is an eigenvector of $\mathcal{H}^{\prime}$ associated with the eigenvalue $r$. We know that $\rho(\mathcal{H}) \leq\|\mathcal{H}\|_{1}=$ $\left\|\mathcal{H}^{\prime}\right\|_{\infty}=r$. Since $r$ is an eigenvalue of $\mathcal{H}^{\prime}$, we get $r \leq \rho\left(\mathcal{H}^{\prime}\right)=\rho(\mathcal{H})$ by the fact that $\mathcal{H}$ and $\mathcal{H}^{\prime}$ have the same spectra of eigenvalues. Therefore, $\rho(\mathcal{H})=r$. From the aforementioned monotonicity of nonnegative matrices with respect to any induced norm, we conclude that $\left\|\mathcal{G}^{m}\right\| \geq\left\|\mathcal{H}^{m}\right\| \Leftrightarrow\left\|\mathcal{G}^{m}\right\|^{\frac{1}{m}} \geq\left\|\mathcal{H}^{m}\right\|^{\frac{1}{m}}$ for all $m \geq$ 1. The last inequality applies due to the fact that the root function is also a monotonic function. By the continuity of the induced matrix norms and the root function for positive real numbers, we have $\mathcal{R}_{0}=\rho(\mathcal{G}) \geq r>0$ in the limit.

Through the model $(\mathcal{M} 3)$ we will demonstrate how we obtain $L$ and $\omega$. Zeroing the right-hand side of 17a), we immediately obtain

$$
S=\frac{\mu N}{\mu+\sum_{\sigma \in 2^{\langle n\rangle} \backslash \emptyset} \frac{\alpha}{N} V_{\sigma}},
$$

where in a neighborhood of $\mathbf{x}_{I}=0$

$$
S=\mu N\left(\frac{1}{\mu}-\frac{1}{\frac{\alpha}{N} \mu^{2}} \sum_{\sigma \in 2^{\langle n\rangle} \backslash \emptyset} V_{\sigma}+\mathcal{O}\left(\left\|\mathbf{x}_{I}\right\|^{2}\right)\right) .
$$

Furthermore, using the result from $17 \mathrm{c}$,

$$
R_{i}=\frac{\gamma}{\nu+\mu} I_{i}
$$

from 17d) we obtain

$$
S_{i}=\frac{\frac{\nu \gamma}{\nu+\mu} I_{i}}{\mu+\sum_{\sigma: \sigma \neq\{i\}} \frac{\alpha}{N} V_{\sigma}},
$$

where in a neighborhood of $\mathbf{x}_{I}=0$

$$
S_{i}=\frac{\nu \gamma}{\nu+\mu} I_{i}\left(\frac{1}{\mu}-\frac{1}{\frac{\alpha}{N} \mu^{2}} \sum_{\sigma: \sigma \neq\{i\}} V_{\sigma}+\mathcal{O}\left(\left\|\mathbf{x}_{I}\right\|^{2}\right)\right) .
$$

As far as the susceptible mosquito compartment is concerned, we have from $17 \mathrm{~g}$

$$
U=\frac{\Lambda}{\eta+\sum_{i \in\langle n\rangle} \frac{\beta}{N}\left(I_{i}+\sum_{\substack{j \in\langle n\rangle \\ j \neq i}} I_{i j}\right)},
$$

where in a neighborhood of $\mathbf{x}_{I}=0$

$$
U=\Lambda\left(\frac{1}{\eta}-\frac{1}{\frac{\beta}{N} \eta^{2}} \sum_{i \in\langle n\rangle}\left(I_{i}+\sum_{\substack{j \in\langle n\rangle \\ j \neq i}} I_{i j}\right)\right.
$$




$$
\left.+\mathcal{O}\left(\left\|\mathbf{x}_{I}\right\|^{2}\right)\right)
$$

We are now substituting (19), (22), and (24) to the equilibrium equations from (17b), (17e), and (17h), respectively, in order to derive the canonical equation. Owing to 17b), we obtain for all $i \in\langle n\rangle$

$$
\begin{aligned}
0 & =I_{i}-\frac{\alpha}{N(\gamma+\mu)} \sum_{\sigma: i \in \sigma} S p_{i}^{\sigma} V_{\sigma} \\
& =I_{i}-\frac{\alpha}{\gamma+\mu} \sum_{\sigma: i \in \sigma}(1 \\
& \left.-\frac{1}{\frac{\alpha}{N} \mu} \sum_{\sigma \in 2^{\langle n\rangle} \backslash \emptyset} V_{\sigma}+\mathcal{O}\left(\left\|\mathbf{x}_{I}\right\|^{2}\right)\right) p_{i}^{\sigma} V_{\sigma} \\
& =I_{i}-\frac{\alpha}{\gamma+\mu} \sum_{\sigma: i \in \sigma} p_{i}^{\sigma} V_{\sigma}+\mathcal{O}\left(\left\|\mathbf{x}_{I}\right\|^{2}\right) .
\end{aligned}
$$

Meanwhile, from (17e) we obtain for all $i, j \in\langle n\rangle$, where $j \neq i$,

$$
\begin{aligned}
0= & I_{i j}-\frac{\alpha}{N(\gamma+\mu)} \sum_{\sigma: j \in \sigma} S_{i} q_{i j}^{\sigma} V_{\sigma} \\
= & I_{i j}-\frac{\alpha \nu \gamma}{N(\gamma+\mu)(\nu+\mu)} I_{i} \sum_{\sigma: j \in \sigma}\left(\frac{1}{\mu}\right. \\
& \left.-\frac{1}{\frac{\alpha}{N} \mu^{2}} \sum_{\sigma: \sigma \neq\{i\}} V_{\sigma}+\mathcal{O}\left(\left\|\mathbf{x}_{I}\right\|^{2}\right)\right) q_{i j}^{\sigma} V_{\sigma} \\
= & I_{i j}+\mathcal{O}\left(\left\|\mathbf{x}_{I}\right\|^{2}\right) .
\end{aligned}
$$

Finally, from $17 \mathrm{~h}$ we obtain for all $\sigma \in 2^{\langle n\rangle} \backslash \emptyset$

$$
\begin{aligned}
0 & =V_{\sigma}-\frac{\beta}{N \eta} U\left(\sum_{i: i \in \sigma} r_{\sigma}^{i} I_{i}+\sum_{j: j \in \sigma} \sum_{i: i \neq j} s_{\sigma}^{i j} I_{i j}\right) \\
= & V_{\sigma}-\frac{\beta}{N \eta}\left(U^{0}-\frac{U^{0}}{\frac{\beta}{N} \eta} \sum_{i \in\langle n\rangle}\left(I_{i}+\sum_{\substack{j \in\langle n\rangle \\
j \neq i}} I_{i j}\right)\right. \\
+ & \left.\mathcal{O}\left(\left\|\mathbf{x}_{I}\right\|^{2}\right)\right)\left(\sum_{i: i \in \sigma} r_{\sigma}^{i} I_{i}+\sum_{j: j \in \sigma} \sum_{i: i \neq j} s_{\sigma}^{i j} I_{i j}\right) \\
= & V_{\sigma}-\frac{\beta U^{0}}{N \eta}\left(\sum_{i: i \in \sigma} r_{\sigma}^{i} I_{i}+\sum_{j: j \in \sigma} \sum_{i: i \neq j} s_{\sigma}^{i j} I_{i j}\right) \\
& +\mathcal{O}\left(\left\|\mathbf{x}_{I}\right\|^{2}\right) .
\end{aligned}
$$

In light of (25), 26), and (27), the canonical equation now has the form

$$
(\operatorname{id~}-\lambda \underbrace{\left(\begin{array}{c|c}
0 & \frac{\mathcal{F}_{12}}{\gamma+\mu} \\
\hline \frac{\mathcal{F}_{21}}{\eta} & 0
\end{array}\right)}_{L}) \mathbf{x}_{I}+\omega\left(\mathbf{x}_{I}, \lambda\right)=0,
$$

where

$$
\lambda=1 \text { and }\|\omega\|=\mathcal{O}\left(\left\|\mathbf{x}_{I}\right\|^{2}\right) .
$$

Observe that $L$ already resembles the next generation matrix $\mathcal{G}$.

Now we are showing that $\mathcal{R}_{0}$ is also the largest eigenvalue of $L$. By using all possible positive eigenvalues $\tau$, we shall decompose $\mathcal{G}-\tau$ id as

$$
\begin{aligned}
& \left(\begin{array}{c|c}
-\tau \text { id } & \frac{\mathcal{F}_{12}}{\eta} \\
\hline \frac{\mathcal{F}_{21}}{\gamma+\mu} & -\tau \text { id }
\end{array}\right) \\
& =\left(\begin{array}{c|c}
-\tau \text { id } & 0 \\
\hline \frac{\mathcal{F}_{21}}{\gamma+\mu} & \mathrm{id}
\end{array}\right)\left(\begin{array}{c|c}
\mathrm{id} & -\frac{1}{\tau} \frac{\mathcal{F}_{12}}{\eta} \\
\hline 0 & -\tau \mathrm{id}+\frac{1}{\tau} \frac{\mathcal{F}_{21}}{\gamma+\mu} \frac{\mathcal{F}_{12}}{\eta}
\end{array}\right)
\end{aligned}
$$

such that

$$
\operatorname{det}(\mathcal{G}-\tau \mathrm{id})=\operatorname{det}\left(-\tau \mathrm{id}+\frac{1}{\tau} \frac{\mathcal{F}_{21}}{\gamma+\mu} \frac{\mathcal{F}_{12}}{\eta}\right),
$$

after taking account of the different dimensions of the identity matrices ids used in the formulation. In the same way as for the matrix $L$, we have

$$
\operatorname{det}(L-\tau \text { id })=\operatorname{det}\left(-\tau \mathrm{id}+\frac{1}{\tau} \frac{\mathcal{F}_{21}}{\eta} \frac{\mathcal{F}_{12}}{\gamma+\mu}\right) .
$$

Therefore, it now follows that

$$
\begin{aligned}
\mathcal{R}_{0} & =\max _{\tau>0}\{\tau: \operatorname{det}(\mathcal{G}-\tau \mathrm{id})=0\} \\
& =\max _{\tau>0}\left\{\tau: \operatorname{det}\left(-\tau \mathrm{id}+\frac{1}{\tau} \frac{\mathcal{F}_{21}}{(\gamma+\mu)} \frac{\mathcal{F}_{12}}{\eta}\right)=0\right\} \\
& =\max _{\tau>0}\left\{\tau: \operatorname{det}\left(-\tau \mathrm{id}+\frac{1}{\tau} \frac{\mathcal{F}_{21}}{\eta} \frac{\mathcal{F}_{12}}{(\gamma+\mu)}\right)=0\right\} \\
& =\max _{\tau>0}\{\tau: \operatorname{det}(L-\tau \mathrm{id})=0\}
\end{aligned}
$$

which leads us to the desired conclusion.

Additionally, the irreducibility test over the matrix $L$ shows that the matrix is indeed reducible. This stems from the fact that $\mathcal{F}_{12}$ contains rows whose entire entries are zero, and so does $\mathcal{F}_{12} /(\gamma+\mu)$. By means of zero rows, there are no ways to go from the vertices indexed by the rows to the other vertices.

\section{Concluding remarks}

An alternative approach to relate the existence of a nontrivial equilibrium (coexistence, boundary or endemic equilibrium) to the basic reproductive number was proposed. The approach was due to transforming the equilibrium equation $f(\mathbf{x} ; \xi)=0$ inherent from an autonomous model into a canonical equation (id $\lambda L) \mathbf{x}_{I}+\omega\left(\mathbf{x}_{I}, \lambda\right)=0$. The basic reproductive number $\mathcal{R}_{0}$ was calculated using the next generation method. Our final result shows that if (i) $\mathcal{R}_{0}$ is the largest eigenvalue 
of $L$, (ii) the matrix $L$ is nonnegative and bounded with respect to all possible values of $\xi$ in some compact set, (iii) $\lambda^{-1}$ is not an eigenvalue of $L$, and (iv) $\mathcal{R}_{0}>1$ is close enough to $\lambda^{-1}$, then there exists a nonnegative nontrivial solution of the canonical equation. Additionally, if the matrix $L$ is irreducible, then there exists a strictly positive nontrivial solution of the canonical equation.

The components of the canonical equation and the basic reproductive numbers as well as their associated eigenvectors for the three models $(\mathcal{M} 1),(\mathcal{M} 2)$ and $(\mathcal{M} 3)$ are given in Table 1 We shall mention that the nonlinear parts $\omega$ for $(\mathcal{M} 2)$ and $(\mathcal{M} 3)$ are too complicated to write but happen to be $\mathcal{O}\left(\left\|\mathbf{x}_{I}\right\|^{2}\right)$ as $\mathbf{x}_{I} \rightarrow 0$. Moreover, the table gives us evidence that the eigenvectors are positive, except that from $(\mathcal{M} 3)$, where the corresponding matrix $L$ is reducible. Moreover, $\mathcal{R}_{0}>1$ does not always mean that $\lambda^{-1}$ (in the three models it is apparently given by 1 ) is not an eigenvalue of $L$. Exclusion of $\lambda^{-1}$ from the spectrum of $L$ remains to be assumed in all the models. Also, we have to make sure that the off- $\mathbf{x}_{I}$ compartments, in the case $(\mathcal{M} 2) \quad S, R, C, U$ and of $(\mathcal{M} 3) S, R_{1}, \ldots, R_{n}, S_{1}, \ldots, S_{n}, U$, in the equilibrium cannot be negative as $\mathbf{x}_{I}$ is nonnegative (or positive). Apparently, they are not based on our calculations, while excluded from our presentation here.

\section{Acknowledgment}

This research is supported by the Indonesia Endowment Fund for Education (LPDP) and partially supported by DAAD through the project Mathematical Models for Dengue Fever (DAAD PPP-Portugal, project ID 57128360). The authors are also grateful to anonymous reviewers for their valuable suggestions.

\section{References}

Aguiar, M., Kooi, B.W., Rocha, F., Ghaffari, P. and Stollenwerk, N. (2013). How much complexity is needed to describe the fluctuations observed in dengue hemorrhagic fever incidence data?, Ecological Complexity 16: 31-40.

Arino, J., Miller, J.M. and van den Driessche, P. (2005). A multi-species epidemic model with spatial dynamics, Mathematical Medicine and Biology: A Journal of the IMA 22(2): 3140.

Cushing, J.M. (1998). An Introduction to Structured Population Dynamics, CBMS-NSF Regional Conference Series in Applied Mathematics, SIAM, Philadelphia, PA.

Gelfand, I. (1941). Normierte Ringe, Mathematiceskii Sbornik 9(51)(1): 3-24.

Golubitsky, M. and Schaeffer, D.G. (1985). Singularities and Groups in Bifurcation Theory, Vol. I, Springer, New York, NY.
Horn, R.A. and Johnson, C.R. (2013). Matrix Analysis, 2nd Ed., Cambridge University Press, New York, NY.

Krasnoselskii, M. and Zabreiko, P. (1984). Geometrical Methods of Nonlinear Analysis, Springer, New York, NY.

Ma, T. and Wang, S. (2005). Bifurcation Theory and Applications, World Scientific Series on Nonlinear Science A, Vol. 53, World Scientific Publishing, Singapore.

Nirenberg, L. (2001). Topics in Nonlinear Functional Analysis, Courant Lecture Notes in Mathematics 6, New York University Courant Institute of Mathematical Sciences, New York, NY.

Ortega, J.M. (1932). Numerical Analysis: A Second Course, SIAM, Philadelphia, PA.

Rabinowitz, P.H. (1971). Some global results for nonlinear eigenvalue problems, Journal of Functional Analysis 7(3): 487-513.

Rabinowitz, P.H. (1977). A bifurcation theorem for potential operators, Journal of Functional Analysis 25(4): 412-424.

van den Driessche, P. and Watmough, J. (2002). Reproduction numbers and sub-threshold endemic equilibria for compartmental models of disease transmission, Mathematical Biosciences 180(1): 29-48.

Wijaya, K.P., Goetz, T. and Soewono, E. (2014). An optimal control model of mosquito reduction management in a dengue endemic region, International Journal of Biomathematics 7(5): 1450056-22.

Wijaya, K.P., Goetz, T. and Soewono, E. (2016). Advances in mosquito dynamics modeling, Mathematical Methods in the Applied Sciences 39(16): 4750-4763.

Zadeh, L.A. and Desoer, C.A. (1963). Linear System Theory: The State Approach, McGraw-Hill, New York, NY.

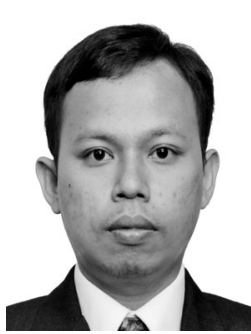

Karunia Putra Wijaya received his BSc and MSc degrees in applied mathematics from the Bandung Institute of Technology, Indonesia, in 2011 and 2012, respectively. He received his $\mathrm{PhD}$ in applied mathematics from the University of Koblenz, Germany, in 2017. His fields of interest include mathematical modeling with differential equations and optimization.

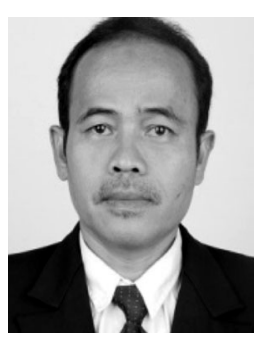

Sutimin graduated from Diponegoro University, Indonesia, in 1989. He received his $\mathrm{PhD}$ in applied mathematics from the Bandung Institute of Technology, Indonesia, in 2017. He is currently the head of the Laboratory of Applied Mathematics at the Department of Mathematics, Diponegoro University. His main fields of interest are mathematical modelling and within host models of HIV infection. 


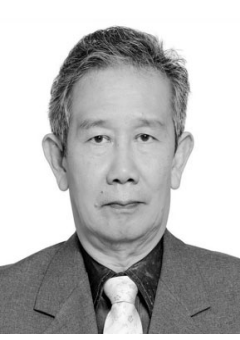

Edy Soewono received his $\mathrm{PhD}$ from Ohio University, USA, in 1988 . He is currently a professor of applied mathematics at the Department of Mathematics, Bandung Institute of Technology, Indonesia. His main field of interest is population dynamics.

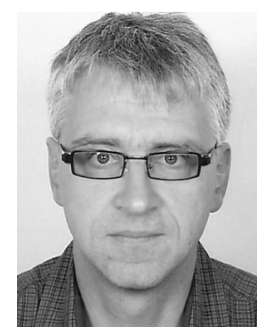

Thomas Götz is a professor of applied mathematics at the University of Koblenz, Germany. $\mathrm{He}$ received his $\mathrm{PhD}$ from the Technical University of Kaiserslautern, Germany, in 2000. He was at the International Center for Theoretical Physics (ICTP) in Trieste, Italy, as a visiting scientist from 2003 to 2004. Since 2005 he has been a member of the Educational Committee of the European Consortium for Mathematics in Industry (ECMI). His main fields of interest are mathematical modeling and numerical methods to simulate and optimize differential equations arising from applied problems.

Received: 2 August 2016

Revised: 2 March 2017

Accepted: 18 April 2017 